\title{
Dialyzer First Use Syndrome
}

National Cancer Institute

\section{Source}

National Cancer Institute. Dialyzer First Use Syndrome. NCI Thesaurus. Code C50532.

A symptom complex characterized by nervousness, chest pain, back pain, palpations, pruritus, and other usually mild symptoms occurring minutes following the initiation of dialysis with a new dialyzer. 